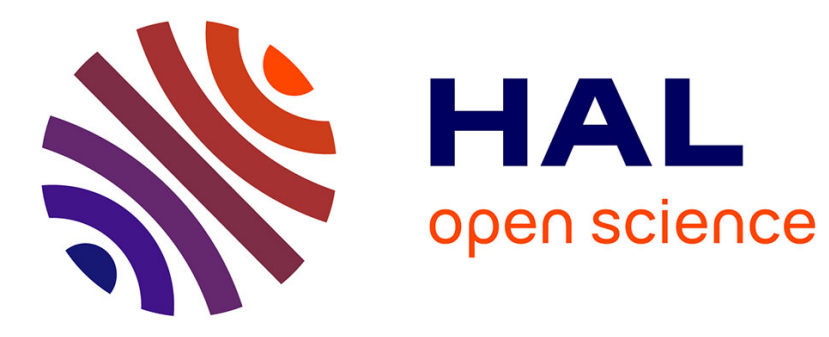

\title{
Scale invariant line matching on the sphere
}

Dieu-Sang Ly, Cédric Demonceaux, Ralph Seulin, Yohan Fougerolle

\section{To cite this version:}

Dieu-Sang Ly, Cédric Demonceaux, Ralph Seulin, Yohan Fougerolle. Scale invariant line matching on the sphere. IEEE International Conference on Image Processing, ICIP'2013, Sep 2013, Melbourne, Australia. hal-00830884

\section{HAL Id: hal-00830884 https://hal.science/hal-00830884}

Submitted on 16 Sep 2013

HAL is a multi-disciplinary open access archive for the deposit and dissemination of scientific research documents, whether they are published or not. The documents may come from teaching and research institutions in France or abroad, or from public or private research centers.
L'archive ouverte pluridisciplinaire HAL, est destinée au dépôt et à la diffusion de documents scientifiques de niveau recherche, publiés ou non, émanant des établissements d'enseignement et de recherche français ou étrangers, des laboratoires publics ou privés. 


\title{
SCALE INVARIANT LINE MATCHING ON THE SPHERE
}

\author{
Dieu-Sang Ly ${ }^{1} \quad$ Cédric Demonceaux ${ }^{2} \quad$ Ralph Seulin $^{2} \quad$ Yohan Fougerolle $^{2}$ \\ ${ }^{1}$ Centre de Morphologie Mathématique, Ecole des Mines de Paris, France \\ ${ }^{2}$ Le2i, UMR CNRS 6306, Université de Bourgogne, Le Creusot, France \\ dieu-sang.ly@mines-paristech.fr
}

\begin{abstract}
This paper proposes a novel approach of line matching across images captured by different types of cameras, from perspective to omnidirectional ones. Based on the spherical mapping, this method utilizes spherical SIFT point features to boost line matching and searches line correspondences using an affine invariant measure of similarity. It permits to unify the commonest cameras and to process heterogeneous images with the least distortion of visual information.
\end{abstract}

Index Terms - Line matching, sphere, hybrid cameras

\section{INTRODUCTION}

It is possible to enhance the field of view (FOV) of conventional perspective cameras by using fish-eye lenses or mirrors of special curvature, multiple cameras looking toward different directions or cameras in rotation. Although these devices provide wide FOVs, they are limited by low resolution or manufacturing complexity. Thus, a parallel utilization of perspective and omnidirectional cameras has become an attractive trend. Along with the increasing deployment of hybrid cameras, feature detection and matching between hybrid images has become an important topic in computer vision. There exist several works on detection and matching of point features between images captured by heterogeneous cameras. Nevertheless, the number of methods dedicated to line features is very limited, particularly in case of hybrid images. We can see in the following literature review that some solutions have been proposed to match lines between perspective images and that only a few ones deal with omnidirectional images. To the best of our knowledge, the only existing method of line matching between images captured by different types of central cameras could be [1]. However, it is constrained to short base-line motion.

Hence, we propose a novel approach of line matching between images captured by different types of cameras, from perspective to omnidirectional ones. The entire algorithm is developed in the spherical space which allows to unify dissimilar imaging devices [2, 3]. Line correspondences are searched through spherical scale-invariant-feature-transform
(SIFT) point features using an affine invariant measure of similarity.

The paper is organized as follows: section 2 provides an overview of related works; the proposed algorithm of line matching is presented in section 3, section 4 shows some experimental results and finally, the paper is concluded in section 5 .

\section{RELATED WORKS}

Only a few methods have been presented in the literature for automatic line matching. The very first algorithms of line matching [4, 5] were based on known multiple view geometry. Bay et al. [6] proposed a solution of line matching based on photometric and topological information: first, line segments are matched using the histograms of line neighborhoods, and then the topological relations of line segments are used to define correct and wrong matches. Using color histograms makes this method less robust to illumination change. Wang et al. [7] developed the Mean - Standard deviation Line Descriptor (MSLD) which is distinctive against rotation, illumination change, image blur, viewpoint change, noise, compression and partial occlusion. The MSLD descriptor is determined by the mean and standard deviation of the histogram built from the neighborhood of each line. Obviously, this descriptor is dependent upon the neighborhoods of line segments and the matching may fail in case of repeated regions such as building facades. Wang et al. [8] introduced a matching approach using line signatures (LS). LS descriptor is computed from the angle and length ratios between lines defined by two end-points, therefore this descriptor is not robust if the end-points are not accurate. Recently, Fan et al. [9] proposed a line matching method based on point correspondences. They proved that it outperformed the LS descriptor in terms of number of correct matches and the MSLD in case of scale change. Kim and Lee [10] search for line correspondences and estimate the epipolar geometry between two images simultaneously using intersecting line pairs in 2D images. In order to deal with poorly structured/textured scene, they combine the geometrical invariance of 3D line intersection and the photometric invariance of 2D line intersection.

Concerning line matching in omnidirectional images, 
Mosaddegh et al. [1] proposed a line matching approach for all central projection cameras. First the rotation between two images is computed from vanishing points of parallel lines or point correspondences. Then lines are matched using the recovered rotation which relates line normals on spherical images. This method is only applicable to short baseline image pairs. Scaramuzza et al. [11] presented a method of vertical line matching in omnidirectional images using the dissimilarity measure between two descriptors, i.e. the Euclidean distance between two associated histograms.

\section{LINE MATCHING IN SPHERICAL SPACE}

\subsection{Detection and matching of spherical SIFT points}

Several methods have been proposed to adapt the SIFT features to omnidirectional images. Whereas most of them apply the classical SIFT to unwrapped omnidirectional images, Cruz-Mota et al. [12] compute the SIFT features in the spherical space, which preserves the spherical affine transformations and processes the visual information without introducing any deformation. The extraction of SIFT features on the spherical image consists of the following steps:

1. Map the original image on the sphere with $(\theta, \varphi)$ parameterization. For instance, images from perspective, catadioptric or fish-eye cameras can be mapped on the sphere using the unified projection model [2, 3] and images captured by Ladybug cameras can be directly used as spherical images.

2. Compute the spherical scale-space representation of the spherical image and then the spherical difference-ofGaussians (DoGs)

3. Extract the local extrema from the DoGs. Each extremum is detected by comparing a point to its 26 neighbors in $3 \times 3$ spherical grids at the current and adjacent scales.

4. Calculate the spherical descriptor of each extracted keypoint. This descriptor is created from orientation histograms of the region around the keypoint.

Once the keypoints and their descriptors are obtained, we can find the matched points between two images using the method proposed by Lowe in [13].

\subsection{Line extraction}

An algorithm of line detection in images captured by catadioptric cameras with paraboloid mirror was proposed in [14]. The extraction processes both original and spherical images as follows:

1. Extract edges in the original image using Canny edge detector

2. Link edge points into chains with a threshold of minimal chain length

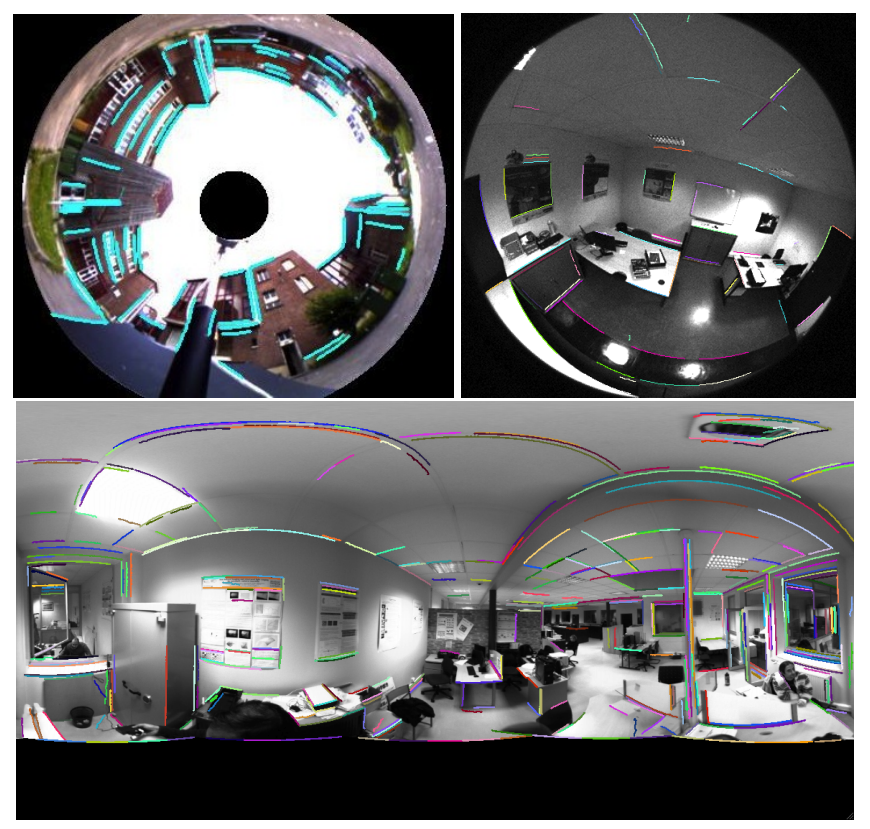

Fig. 1. Line detection in wide-angle images

3. Split chain which does not correspond to a unique line into sub-chains as each chain after the second step may contain the projection of different 3D lines

4. Merge sub-chains which belong to the same line as subchains after the third step may be segments of a single line

We have extended this algorithm to other wide-angle cameras such as central catadioptric, fish-eye and Ladybug cameras as illustrated in figure 1

\subsection{Line matching through point correspondences}

Once point correspondences and line features are available in two images, we proceed to match these line sets using algorithm 11. In this algorithm, the similarity between two lines $\mathbf{l}_{1}$ and $\mathbf{l}_{2}$ is computed from point correspondences in their neighborhood (figure 2(b)) by algorithm 2. This similarity is measured as the maximal median of the individual similarities calculated from two pairs of matched points $\left\{\mathbf{p}_{m}, \mathbf{q}_{m}\right\}$ and $\left\{\mathbf{p}_{n}, \mathbf{q}_{n}\right\}$ as follows:

$$
\operatorname{Sim}\left(\left\{\mathbf{p}_{m}, \mathbf{q}_{m}\right\},\left\{\mathbf{p}_{n}, \mathbf{q}_{n}\right\}\right)=e^{-\left\|D\left(\mathbf{p}_{m}, \mathbf{p}_{n}, \mathbf{l}_{1}\right)-D\left(\mathbf{q}_{m}, \mathbf{q}_{n}, \mathbf{l}_{2}\right)\right\|}
$$

where $D$ is obtained from the ratio of distances from two points to a line. As we work in spherical space, the classical point-line distance becomes the geodesic distance as illustrated in figure 2(a). The $2 \mathrm{D}$ point is projected to the spherical point $\mathbf{p}$ in blue and the $2 \mathrm{D}$ line segment $\mathbf{l}$ is projected to the green arc lying on a great circle associated with its normal $\mathbf{n}$. 

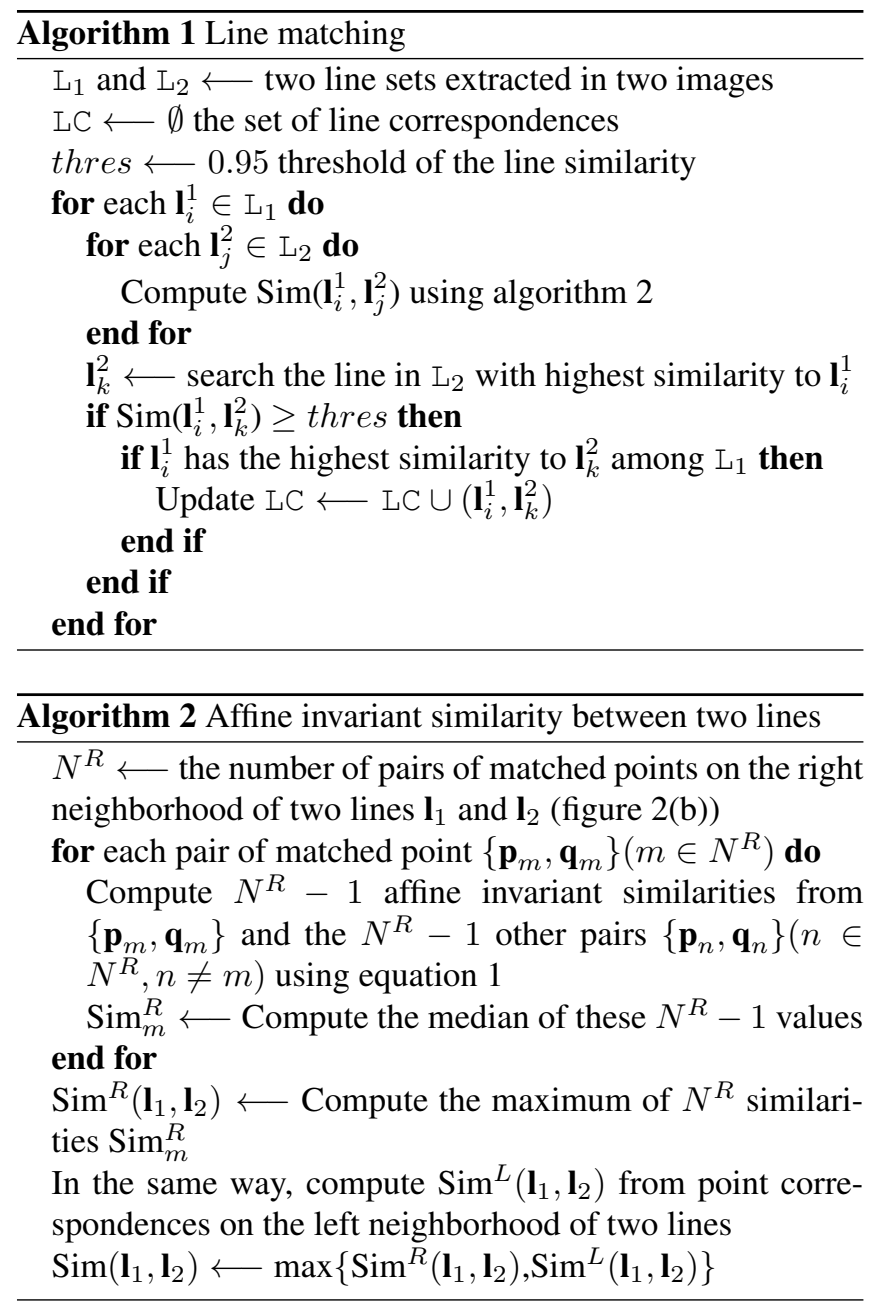

The geodesic distance between the point and the line segment is the length of the red arc. This length is proportional to the scalar product between the spherical point $\mathbf{p}\left(x_{\mathbf{p}}, y_{\mathbf{p}}, z_{\mathbf{p}}, 1\right)$ and the normal $\mathbf{n}\left(x_{\mathbf{n}}, y_{\mathbf{n}}, z_{\mathbf{n}}, 0\right)$. Therefore, $D$ can be calculated by

$$
D\left(\mathbf{p}_{m}, \mathbf{p}_{n}, \mathbf{l}\right)=\frac{\mathbf{n}^{\top} \mathbf{p}_{m}}{\mathbf{n}^{\top} \mathbf{p}_{n}}
$$

As long as these two points located in the neighborhood and coplanar with the line, $D$ is affine invariant [9]. Therefore, in order to obtain an affine invariant similarity, the point correspondences used for line matching must be coplanar with the line. This condition is normally satisfied as lines detected in images usually correspond to edges of 3D surfaces where we can obtain coplanar points.

\section{EXPERIMENTAL RESULTS}

The proposed approach of line matching is evaluated on a pair of images captured by a Ladybug camera as illustrated in figure 3 Firstly, point features are extracted and matched using the method presented in section 3.1. The implementation of

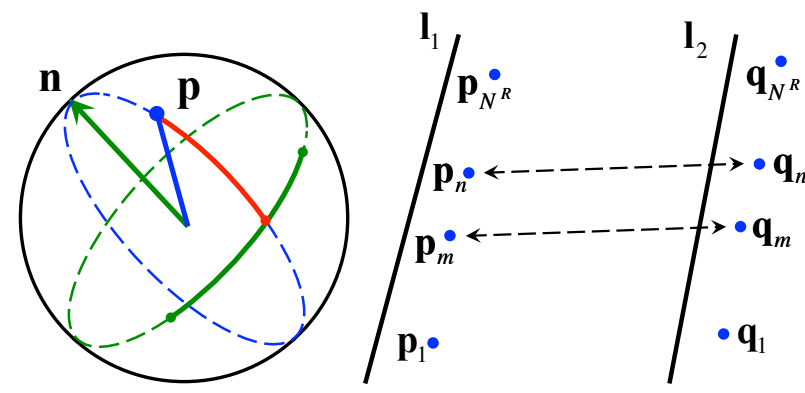

(a)

(b)

Fig. 2. (a) Point and line projection with its normal on sphere - (b) Two lines with point correspondences

point detection and matching together with Ladybug images is publicly available [12]. From 624 and 599 spherical SIFT points extracted in the first and second images respectively, we obtained 59 point correspondences (plotted in red dots). In addition, 255 and 217 lines were detected in these images by the technique in section 3.2 and are shown in random colors in figure 3 The result of line matching by our proposed algorithm in section 3.3 is presented in figure 4 Each pair of matched lines is displayed in the same color. Each row in this figure shows the similar angle of view in two images where we can find both correct and incorrect line matches. Whether a line match is correct or not is verified manually. There are 15 correct matches among 21 matches in total. Most of the correctly matched lines locate in the middle of image (doors, posters) and most of the incorrect matches are found on the ground. The incorrect line matching is due to wrong point matches or similar ratios of point-line distances.

\section{CONCLUSIONS}

The paper proposed a novel approach of line matching in spherical space. Given two spherical images, line matching can be achieved through three main steps: searching for point correspondences using spherical SIFT features, detecting lines and matching two line sets using an affine invariant similarity based on the ratio of point-line distances. This method can be applied to any camera which is equivalent to a central imaging system. As most of the commonly used cameras such as perspective, catadioptric, fish-eye and Ladybug ones can be modeled as spherical cameras, this can be a unified approach to search for line correspondences among different kinds of cameras. Potential applications are extrinsic calibration of a hybrid camera network, motion estimation using dissimilar types of cameras, etc. From the observation in the experimental results, one of the perspectives of this work is to encode the line orientation into the matching algorithm in order to improve the solution. Experiments with heterogeneous images would also be useful to evaluate this method. 


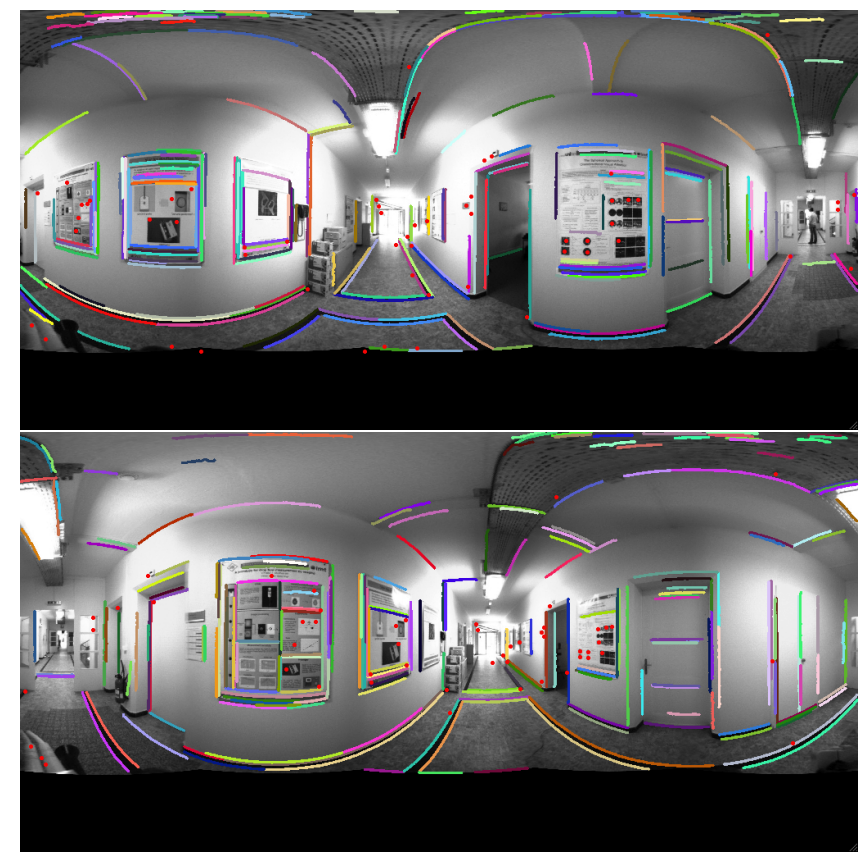

Fig. 3. Point correspondences and detected lines in two Ladybug images

\section{Acknowledgments}

This work has been funded by Le Conseil Régional de Bourgogne et Les Fonds Européens de Développement Régionaux (FEDER)

\section{REFERENCES}

[1] S. Mosaddegh, D. Fofi, and P. Vasseur, "A generic method of line matching for central imaging systems under short-baseline motion," in International Conference on Image Analysis and Processing, 2009, pp. 939-948.

[2] C. Geyer and K. Daniilidis, "A unifying theory for central panoramic systems and practical implications," in European Conference on Computer Vision, 2000, pp. II: 445-461.

[3] X.H. Ying and Z.Y. Hu, "Can we consider central catadioptric cameras and fisheye cameras within a unified imaging model," in European Conference on Computer Vision, 2004, pp. I: 442455.

[4] R.I. Hartley, "A linear method for reconstruction from lines and points," in International Conference on Computer Vision, 1995, pp. 882-887.

[5] C. Schmid and A. Zisserman, "Automatic line matching across views," in IEEE Conference on Computer Vision and Pattern Recognition, 1997, pp. 666-671.

[6] H. Bay, V. Ferrari, and L.J. Van Gool, "Wide-baseline stereo matching with line segments," in IEEE Conference on Computer Vision and Pattern Recognition, 2005, pp. I: 329-336.

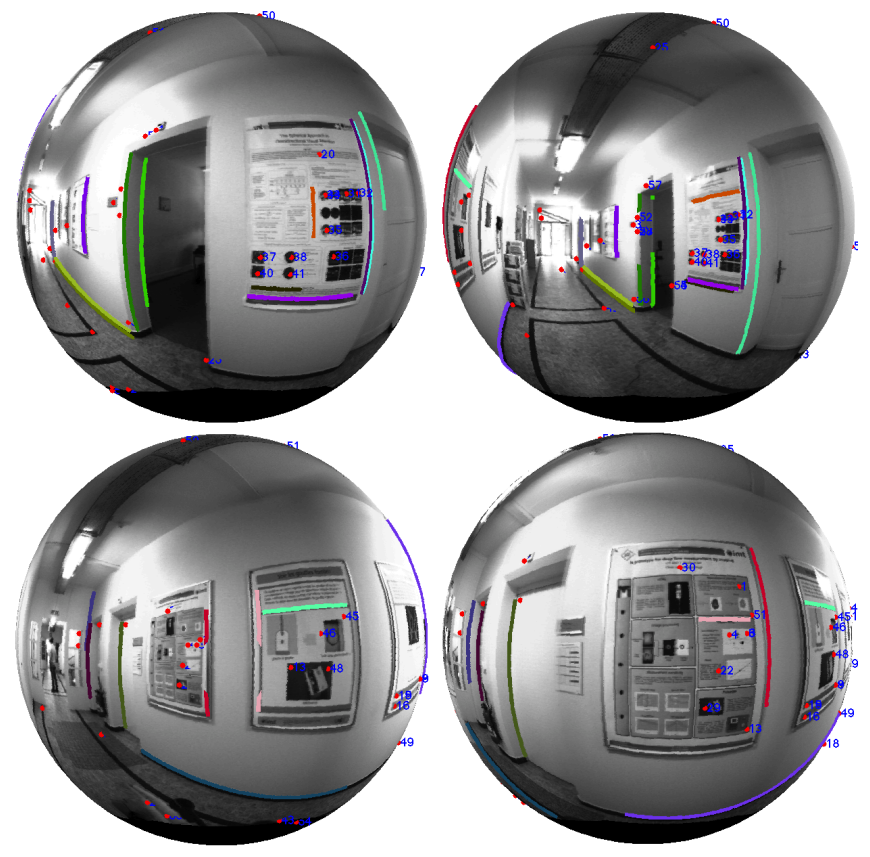

Fig. 4. Line matching between two Ladybug images

[7] Z.H. Wang, F.C. Wu, and Z.Y. Hu, "Msld: A robust descriptor for line matching," Pattern Recognition, vol. 42, no. 5, pp. 941-953, May 2009.

[8] L. Wang, U. Neumann, and S. You, "Wide-baseline image matching using line signatures," in International Conference on Computer Vision, 2009, pp. 1311-1318.

[9] B. Fan, F.C. Wu, and Z.Y. Hu, "Line matching leveraged by point correspondences," in IEEE Conference on Computer Vision and Pattern Recognition, 2010, pp. 390-397.

[10] H.W. Kim and S. Lee, "Simultaneous line matching and epipolar geometry estimation based on the intersection context of coplanar line pairs," Pattern Recognition Letters, vol. 33, no. 10, pp. 1349-1363, July 2012.

[11] Davide Scaramuzza, Roland Siegwart, and Agostino Martinelli, "A robust descriptor for tracking vertical lines in omnidirectional images and its use in mobile robotics," International Journal of Robotics Research, vol. 28, pp. 149-171, February 2009.

[12] J. Cruz Mota, I. Bogdanova, B. Paquier, M. Bierlaire, and J.P. Thiran, "Scale invariant feature transform on the sphere: Theory and applications," International Journal of Computer Vision, vol. 98, no. 2, pp. 217-241, June 2012.

[13] D.G. Lowe, "Distinctive image features from scale-invariant keypoints," International Journal of Computer Vision, vol. 60, no. 2, pp. 91-110, November 2004.

[14] J.C. Bazin, C. Demonceaux, P. Vasseur, and I. Kweon, "Motion estimation by decoupling rotation and translation in catadioptric vision," Computer Vision and Image Understanding, vol. 114, no. 2, pp. 254-273, February 2010. 